\title{
Values and Limits of Telemedicine: a Case Report
}

\author{
Nikos Pappan ${ }^{1}$ (D) $\cdot$ Raed Benkhadra ${ }^{1}$ - Danielle Papincak ${ }^{1} \cdot$ Kameron Ashker $^{1} \cdot$ Jeffrey Uchin $^{2} \cdot$ Nadeera Sidique $^{3}$. \\ Zahra Pirani $^{3}$. Patrice Clemenza ${ }^{4}$
}

Accepted: 22 December 2020 / Published online: 4 January 2021

(C) The Author(s), under exclusive licence to Springer Nature Switzerland AG part of Springer Nature 2021

\begin{abstract}
In the era of a pandemic, the utilization of telemedicine is growing at a rapid speed. This new and necessary adaption in medicine is a threat to the basics of medicine which include the physical exam. A 72-year-old woman presents for a 1-week history of cervical neck discomfort. The patient was found to be febrile with initial physical exam nonrevealing due to patient preference of not taking off hospital gown. After blood cultures grew group A beta-hemolytic streptococcus and a computed tomography scan of the abdomen and pelvis with contrast demonstrated subtle bilateral renal hypodensities suggesting possible septic emboli, a more thorough physical exam was sought out which revealed a large rodent ulcer which the patient had been hiding from her family for 2 years. Transthoracic echocardiography was done which demonstrated a vegetation on the mitral valve confirming the diagnosis of endocarditis. The source of infection was the ulcer which was biopsied and found to be basal cell carcinoma. We present a unique case of endocarditis that was reliant on the physical exam to reveal the source of infection which was a rodent basal cell carcinoma ulcer. This case reminds physicians that at the forefront of telemedicine, the physical exam should not be forgotten.
\end{abstract}

Keywords Basal cell carcinoma $\cdot$ Endocarditis $\cdot$ Mitral valve vegetation $\cdot$ Physical exam

\section{Background}

Medicine is a constantly evolving science with new technologies and modalities being created every year. With more sensitive testing being created, physicians often find themselves questioning the sensitivity of the physical exam compared to modern medicine modalities. Some conclusions state the physical exam can be just as sensitive, cost-saving, and more therapeutically touching or warming to patients [1].

This article is part of the Topical Collection on Medicine

Nikos Pappan

Nikospappan@gmail.com

1 Department of Internal Medicine, Internal Medicine Institute, Allegheny General Hospital, 320 E North Ave., Pittsburgh, PA 15212, USA

2 Pathology Department, Allegheny General Hospital, 320 E North Ave., Pittsburgh, PA 15212, USA

3 Drexel University Medical School, 2900 West Queen Lane, Philadelphia, PA 19129, USA

4 Duquesne University, 600 Forbes Ave., Pittsburgh, PA 15282, USA
It is important during these times of social distancing and limiting viral exposure that physicians do not forget the foundation of their physical exam skills starting with inspection. Physicians will eventually have to adapt the physical exam with this new onset of telemedicine but when appropriate should resort back to our original teachings consisting of inspection, palpation, and auscultation. In view of this, we demonstrate a unique case that exemplifies the importance of the physical exam during this modern era of telemedicine.

\section{Case Presentation}

A 72-year-old woman with no significant past medical history presented to the hospital for 1 week of cervical neck discomfort, mild lower back discomfort, and right hip discomfort. Before the presentation, she went to medical express for examination. At that time, she was given prednisone and Flexeril for suspected sciatica based on history and physical exam revealing left-sided buttocks pain shooting down the left leg. Following this treatment, the pain persisted, and the patient started to have chills, sweats, and subjective fevers several days after that visit. 
At the presentation to the hospital, the patient had a temperature of $102.8 \mathrm{~F}$ and tachycardia but was normotensive stable. Physical exam was grossly benign initially, but the patient refused to put on a hospital gown because she was cold and shivering, therefore not allowing full-body inspection. Labs were consistent with a normal white cell count, mild transaminitis, and elevated procalcitonin; all other labs were unremarkable. A computed tomography scan of the abdomen and pelvis with contrast demonstrated subtle bilateral renal hypodensities suggesting possible septic emboli (Fig. 1). Blood cultures were taken, and the patient was put on broadspectrum antibiotics which included vancomycin and cefepime.

The following day, the patients' blood cultures grew group A beta-hemolytic streptococcus. In-depth discussion of these new results and explanation of the necessity of the physical exam strengthen the patient-physician relationship, after which she consented to be examined. A full physical exam with skin inspection revealed right upper back/posterior shoulder $10 \mathrm{~cm} \times$ $4 \mathrm{~cm}$ ulcerated plaque (Fig. 2), a lesion that the patient confessed she had been hiding from her family for 2 years.

Due to the suspicion of endocarditis with potential showering of septic emboli to the kidney, a transthoracic echocardiography was done which did not reveal any valvular vegetations. For further evaluation, a transesophageal echocardiogram was pursued which revealed a hypodensity on the anterior mitral valve suggestive of a vegetation (Fig. 3). The patient was then put on intravenous cefazolin for 6 weeks for the treatment of infective endocarditis. A skin punch biopsy was performed on the rodent ulcer which revealed basal cell carcinoma (Fig. 4). The patient was advised to follow-up with dermatology for Mohs surgery.

\section{Discussion}

In this case, we present a patient who was diagnosed with infective endocarditis from an ulcerated basal cell carcinoma

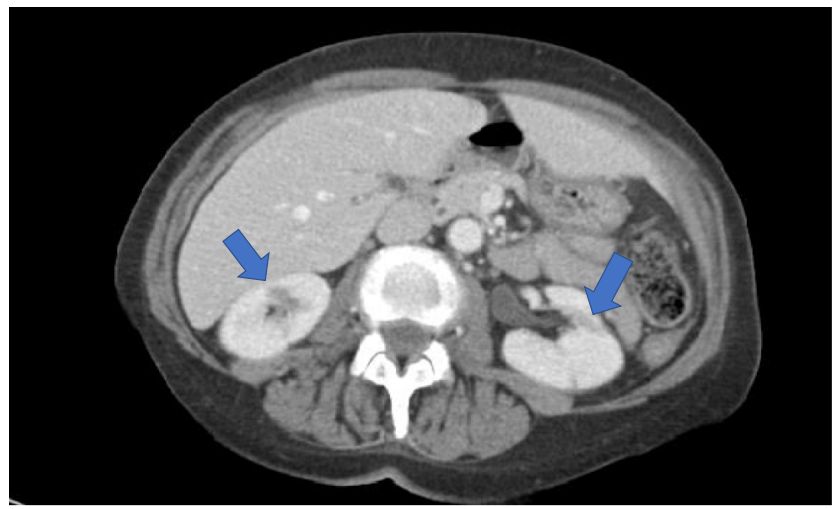

Fig. 1 Computed tomography scan of the abdomen and pelvis with intravenous contrast showing renal hypodensity and subtle bilateral peripheral/subcortical hypodense zones (arrows)

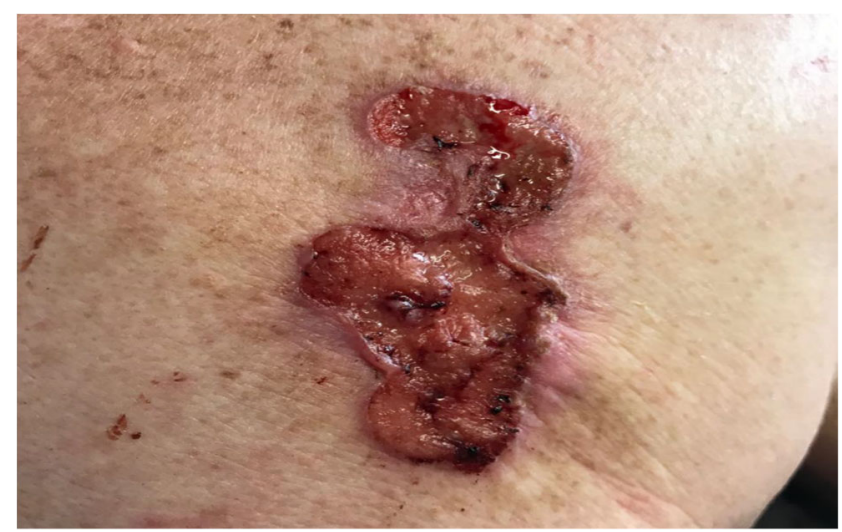

Fig. 2 Rodent ulcer/large basal cell carcinoma. Right upper back/ posterior shoulder $10 \mathrm{~cm} \times 4 \mathrm{~cm}$ ulcerated translucent pink plaque mass with irregular, erythematous, heaped up margins and serosanguinous drainage

wound. The patient presented with fever, renal septic emboli (suggesting vascular phenomenon), blood cultures growing group A beta-hemolytic streptococcus (an uncommon cause of infective endocarditis), and vegetation on transesophageal echocardiogram. This exemplifies 1 major and 3 minor Duke's criteria confirming the diagnosis of endocarditis [2]. In pursuit of an infection source on a patient with no risk factors such as intravenous drug use, one must rely heavily on the physical exam, starting with inspection. Due to patient preference, full-body inspection was initially not persuaded. However, establishing a good patient-physician relationship pursued the patient to allow full inspection revealing a unique infectious source, an ulcerated basal cell carcinoma.

Since the recent outbreak of the coronavirus pandemic, the medical field has been trying to adapt and transition medicine into a new era involving telemedicine. This new style of medicine will undoubtedly change the basics of the physical exam. New technologies have to be made such as electronic stethoscopes, tele-ophthalmoscopes, and video-otoscopes [3]. Even

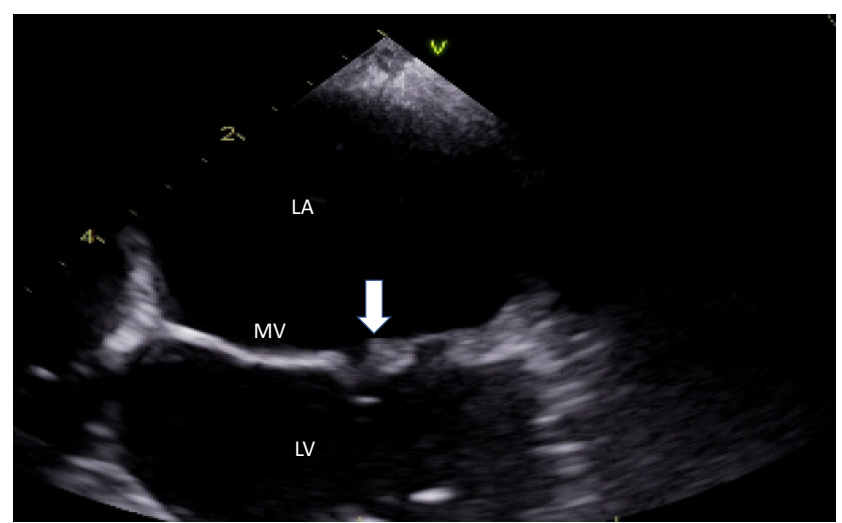

Fig. 3 Mitral valve vegetation. Transesophageal echocardiogram showing zoomed-in long axis view with the left ventricle (LV) on the bottom and left atrium (LA) on the top. The mitral valve (MV) between the two chambers demonstrates a small and non-mobile vegetation (arrow) present on the anterior leaflet 


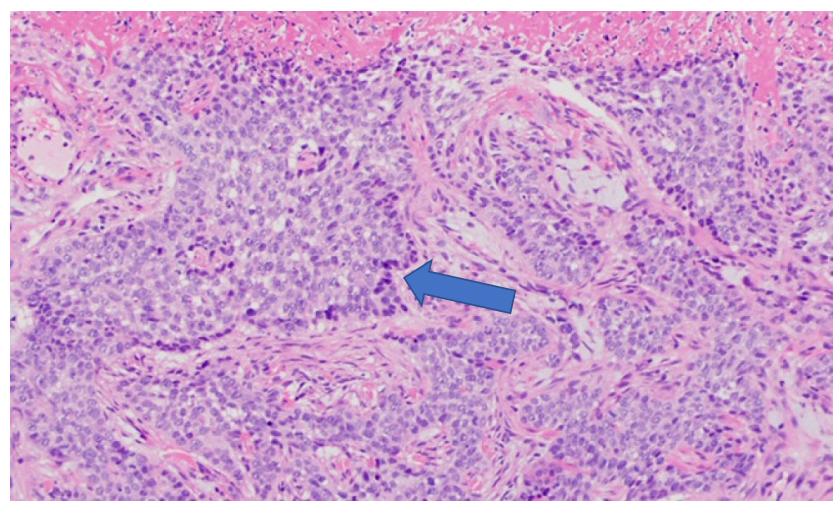

Fig. $4 \times 200$ magnification H\&E stain of skin biopsy. Basaloid tumor cells forming irregularly shaped islands, with those tumor cells focally exhibiting a bit of peripheral palisade (blue arrow) and some localized retraction artifact away from the surrounding inflamed, fibrotic tumor stroma

in the hospital inpatient setting, for example, distracting personal protective equipment (PPE) can potentially alter or decrease the efficacy of the auscultation during the physical exam. Also, many institutes created inpatient "e-consults" for consultant teams to limit exposure and preserve PPE which eliminates the physical exam all together [4]. All of these are necessary measures but could introduce the risk of missing physical exam findings that may be crucial to a correct and timely diagnosis as demonstrated by this unique case.

\section{Conclusion}

This case encompasses many aspects of medicine and validates the importance of the physical exam through a unique presentation of endocarditis with an infected basal cell carcinoma ulceration as the bacterial source. In the era of telemedicine, it is difficult to stick to the basics of medicine but when given the opportunity, it is imperative to perform a thorough physical exam for accuracy of clinical diagnosis and better patient outcomes.
Authors' Contributions Nikos Pappan: drafting and revising the original manuscript. Raed Benkhadra, Danielle Papincak, and Kameron Ashker: final revisions and approval of the final manuscript. Nadeera Sidique, Zahra Pirani, and Patrice Clemenza: figure editing and figure legend drafting. Jeffrey Uchin: pathology figure editing and figure legend drafting.

Data Availability Not applicable

\section{Compliance with Ethical Standards}

Conflict of Interest The authors declare that they have no conflict of interest.

Ethics Approval This article does not contain any research studies with human participants or animals performed by any of the authors.

Consent to Participate This article does not contain any research studies with human participants or animals performed by any of the authors.

Consent for Publication All authors give consent to publish.

Code Availability Not applicable

\section{References}

1. Fitzgerald FT. Physical diagnosis versus modern technology. A review. West J Med. 1990;152(4):377-82.

2. Durack DT, Lukes AS, Bright DK, Service DE. New criteria for diagnosis of infective endocarditis: utilization of specific echocardiographic findings. The American Journal of Medicine. 1994;96(3): 200-9.

3. Weinstein RS, Krupinski EA, Doarn CR. Clinical examination component of telemedicine, telehealth, mHealth, and connected health medical practices. Medical Clinics of North America. 2018;102(3): 533-44.

4. Gadzinski AJ, Andino JJ, Odisho AY, Watts KL, Gore JL, Ellimoottil C. Telemedicine and eConsults for Hospitalized Patients During COVID-19. Urology. 2020;141:12-4. https://doi. org/10.1016/j.urology.2020.04.061.

Publisher's Note Springer Nature remains neutral with regard to jurisdictional claims in published maps and institutional affiliations. 